\title{
It's Food Fight! Designing the Chef's Hat Card Game for Affective-Aware HRI
}

\author{
Pablo Barros, Alessandra Sciutti \\ Cognitive Architecture for \\ Collaborative Technologies Unit \\ Istituto Italiano di Tecnologia \\ Genova, Genova, Italy
}

\author{
Anne C. Bloem, Inge M. Hootsmans, \\ Lena M. Opheij, \\ Romain H.A. Toebosch \\ Department of Industrial Design \\ Eindhoven University of Technology \\ Eindhoven, The Netherland
}

\author{
Emilia Barakova \\ Department of Industrial \\ Design Eindhoven University \\ of Technology Eindhoven, \\ The Netherland \\ e.i.barakova@tue.nl
}
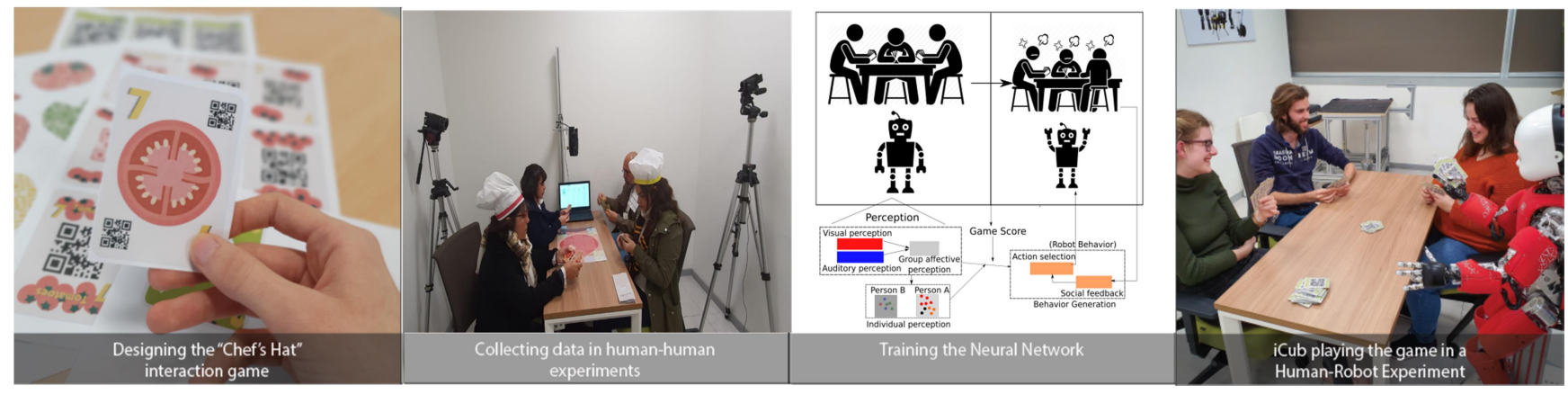

Figure 1: The proposed roadmap for an affective-aware HRI platform: Developing the Chef's Hat card game, collecting the behavioral measures during the game, designing learning models for social robots, and evaluating the game scenario within human-robot interaction.

\begin{abstract}
This paper describes the design of an interactive game between humans and a robot that makes it possible to observe, analyze, and model competitive strategies and affective interactions with the aim to dynamically generate appropriate responses or initiations of a robot. We apply an iterative design process that applied several pilot evaluations to define the requirements for the game with a theme, mechanics, and rules that motivate a choice between competition and cooperation and provokes emotional reactions even after subsequent games. Also, the game is designed to be easily understood by humans and unambiguously interpreted by machines. Overall, we aim to make the Chef's Hat card game a standard platform for the development of competitive and emotionally aware agents and enable embodied interaction between multiple humans and robots.
\end{abstract}

\section{CCS CONCEPTS}

- HCI design and evaluation methods - Interaction design process and methods - Empirical studies in collaborative and social computing

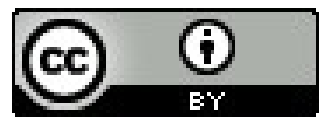

This work is licensed under a Creative Commons Attribution International 4.0 License.

HRI '21 Companion, March 8-11, 2021, Boulder, CO, USA.

(C) 2021 Copyright held by the owner/author(s).

ACM ISBN 978-1-4503-8290-8/21/03. https://doi.org/10.1145/3434074.3447227

\section{KEYWORDS}

Affective Computing; Interaction design in HRI; Games as enablers of HRI

\section{ACM Reference format:}

Pablo Barros, Alessandra Sciutti, Anne C Bloem, Inge M Hootsmans, Lena M Opheij, Romain HA Toebosch, Emilia Barakova, 2021. In Companion of 2021 ACM/IEEE International Conference on Human-Robot Interaction (HRI'21 Companion), March 8-11, 2021, Boulder, CO, USA. ACM, NY, NY, USA, 5 pages. https://doi.org/10.1145/3434074.3447227

\section{Introduction}

Associating perception and action within a continuous adaptation mechanism could improve the way social robots interact and are accepted in real-world social scenarios [5]. An affective architecture, developed in the framework of cognitive computation, is the focus of several researchers over the past decade $[2,6,10]$. Modulating behavior based on affect perception has been explored in human-robot interaction [8]. Most of the existing solutions, however, developed social interaction strategies based on emotion contagion [12] by mirroring the perceived emotion or by relying upon simple decision trees for generating behavior [11]. Such solutions are suited for the simple interaction scenarios in which they were deployed.

Designing, implementing, and deploying realistic interaction scenarios, where competitive and affective information is incorporated into the response of the robot is one of the 
problems that must be solved before the deployment of robots in real-life applications. Previously, a game was designed to favor one and disfavor another player in a multiplayer interaction that targeted monitoring the changes in social interactions and preventing conflicts [1]. This game was designed to provoke spontaneous emotional reactions and thus expressions in players [1], and even the emotional disposition lasted until the next instance of this repetitive game that took place every two weeks between the same players [7]. The game was consequently deployed on a virtual agent that was able to assume strategies of being a cooperative or egoistic partner (i.e by choosing to react cooperatively on the detected cooperative action of the partner) [14].

Barros et al. [4] proposed a social interaction scenario, based on a multiple-players game UNO, to be used in physical affectiveaware Human-Robot Interaction. This game, however, does not allow for a change of hierarchy and play strategy of all players, including the robot, so the emotional involvement can remain high during multiple play occurrences.

To alleviate this shortcoming, in the current study The Chef's Hat is designed to guarantee that the display of affective behavior and its recognition by a robot as a natural and integral part of the game dynamics, ensure emotional involvement during multiple play instances, and necessitate a change of strategy of the players In the iterative design process, we first explored the social dynamics of human players. The integration of robots into the game, as active players, is of fundamental importance. To facilitate that, we included elements in the game's requirements and final mechanics that allow robots to have an estimation of the game status and the person's social behavior and play without disturbing the game flow. As the game is intended to enforce teaming between the players, the occurrence of human-robot teaming behavior is an important part of our game design decisions. The proposed game scenario provides a platform where different learning systems for affective perception, decision making, and behavior generation can be integrated, deployed, and evaluated properly and in an ecologically valid interaction.

\section{Design of The Chef's Hat Game}

\subsection{Interaction Requirements}

The card game was designed to satisfy the following requirements:

1. R1 - The game should elicit a multitude of affective behaviors that the robot can properly understand and model.

2. R2 - The game should be playable without the need for verbal expressions as part of the game mechanics to facilitate affective understanding and easy processing by the robot as robots have difficulties in understanding affect in the semantics of human speech.

3. R3 - The game should provide the possibility of creating strategies based on affective bonding between the players. This way, interacting with other players through the game should be part of the game's natural flow.
4. R4 - The game should have specific mechanics that, when used, cause affective reactions in the players.

5. R5 - The game should be easy to follow and to understand, having clear turns between the players. The number of actions to be made should be small and easy to process. This way, the limitations of the robot regarding decisionmaking, behavior display, and processing time will be reduced.

6. R6 - The game status and players' behavior should be easy to track and monitor using automatic mechanisms (cameras and microphones). This way we can create a knowledge repository to leverage the learning of gaming strategies and behavior understanding by the robot.

7. R7 - The game should give players enough opportunity to interact with all other players through game-mechanics. Actions taken should not only affect the next player in the game, as this could limit both the social and competitive strategies.

\subsection{Pilot test to identify effective features for the new game}

Taking the requirements from Subsection 2.1 into consideration, the design proceeded by evaluating common card games that are well-known to persons from different cultural backgrounds: e.g. UNO, Great Dalmuti, Quartet, Skipbo, and Shithead. These games were chosen since they address a set of requirements 1-7.

Table 1: Summary of which requirements are addressed by each of the tested games.

\begin{tabular}{cccccccc}
\hline Game & R1 & R2 & R3 & R4 & R5 & R6 & R7 \\
\hline UNO & $\checkmark$ & & & $\checkmark$ & $\checkmark$ & $\checkmark$ & \\
Great Dalmuti & $\checkmark$ & $\checkmark$ & $\checkmark$ & & $\checkmark$ & $\checkmark$ & $\checkmark$ \\
Quartet & $\checkmark$ & & $\checkmark$ & & & & $\checkmark$ \\
Skipbo & & $\checkmark$ & & & & $\checkmark$ & \\
Shithead & & $\checkmark$ & & & $\checkmark$ & $\checkmark$ & \\
\hline
\end{tabular}

We performed a pilot test with several rounds of experiments with four participants playing each of these games listed in the first column of Table 1 . We video-recorded and analyzed the behavior of all the players during the game. The behavior observation was carried out by four observers who were previously trained (by education) to evaluate user interactions. All observers scored the games for the requirements 1-7 independently, with a very high interrater agreement.

The analysis showed that popular games, such as UNO and Great Dalmuti encompassed most of the defined requirements, but not all. As discussed in our previous work [4], the possibility of causing adverse affective reactions throughout the gameplay was very present in UNO, but not in the other games. The disadvantage of UNO game was the use of many words and the lack of play elements that encourage bonding throughout the entire gameplay. Great Dalmuti, and UNO were taken as inspiration for creating the new card game that will fit the experimental purpose. The new game presents very simple game mechanics, which is easy to be understood and followed, similar to UNO, but without limiting strategic options as it is composed of different rounds with a continuing score. Furthermore, the 
hierarchy in the gameplay creates interesting group dynamics as well as opportunities for both competitive and cooperative play.

\subsection{Design of physical components of the game}

We started the design process of the Chef's Hat card game taking Great Dalmuti as inspiration. To reduce the feeling of a controlled experiment and make the game feel more like a commercially available card game, a theme was chosen and incorporated throughout all game components. We chose the Kitchen theme, where players compete to become the chef of a pizza restaurant. This theme has been chosen since it can incorporate hierarchy while kitchen interactions are easy to follow for most people. Next to this, an important part of the design of the game components was dictated by the game-state monitoring, necessary for the inclusion of a robot player - a single top-view camera should be used to monitor the entire game-state, so a robot player can make decisions based on analyzing a single image.

The ingredient cards, illustrated in Figure 2, needed to be easily recognizable both when played on the playing field and when exchanged among players at the start of the Shift. In addition to the pictures of the cooking ingredients, QR- codes were added so the robot player can easily be included as an interaction partner.

The cards are to be placed on the playing field, illustrated in Figure 3. While testing the game, a recurrent problem was that players did not play their cards one by one, which made it difficult for the camera to recognize what was played. To counter this, we redesigned the playing field to have 11 different marked places on the pizza, in which players should place their cards.

Role cards were designed to make it easy to see which player has a certain role. The role cards have additional functionality to work as a special action card when turned over. To increase the engagement (and possibly competitiveness), it was opted to also have physical role-related attributes: a Chef's Hat, a Sous-Chef's Hat, a Waiter's bowtie, and a Dishwasher's cloth. The attributes were color-coded per role (see Figure 4).

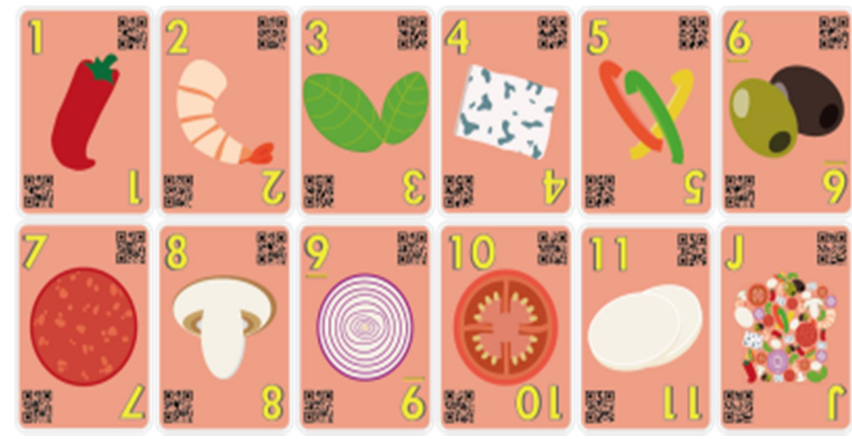

Figure 2: Ingredient and the Joker cards, with their corresponding face number. The lower the number, the rarer the card.

\subsection{Game Mechanics of The Chef's Hat Game}

The game has a role-based hierarchy in a kitchen context: each player can either be a Chef, a Sous-Chef, a Waiter, or a Dishwasher. The players get 17 cards, and each player tries to be the first to get rid of their ingredient which will promote the player to the position of a Chef. This happens for multiple rounds or shifts until the first player reaches 15 points. During every Shift there are three phases: (1) Start of the Shift; (2) Making Pizzas; (3) End of the Shift.

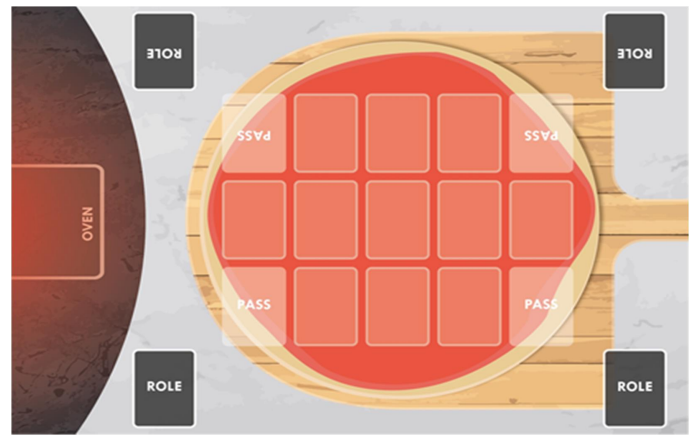

Figure 3: Playing field where the cards are placed, representing a pizza board.
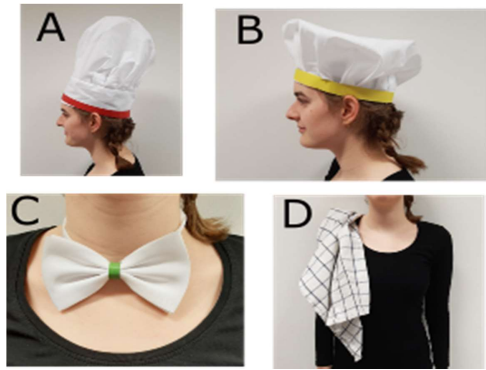

Figure 4: Physical attributes used for each role: A - Chef, B -Sous-Chef, C - Waiter, D - Dishwasher.

At the start of the Shift, all cards are divided among the four players. Then, the Dishwasher has to give the two cards with the highest values to the Chef, who in return gives back two cards of their liking. The Waiter must give their lowest- valued card to the Sous-Chef, who in return gives one card of their liking. If a player has two Jokers at the start of the Shift, they can choose to play their special action: in the case of the Dishwasher this is "Food Fight" (the hierarchy is inverted), in the case of the other roles it is "Dinner is served" (there will be no card exchange during that the Shift).

Then, the making of the pizzas starts. The person who possesses a Golden 11 card may start making the first pizza of the Shift. A pizza is prepared when ingredient cards are played on the pizza base on the playing field. A pizza is done when no one can (or wants to) put on any ingredients anymore. The rarest cards have the lowest numbers. A player can play cards by laying down their ingredient cards on the pizza base. To play the cards, they need to be rarer (i.e. lowest face values) than the previously 
played cards. The ingredients are played from highest to the lowest number, so from 11 to 1 . Players can play multiple copies of an ingredient at once, but always have to play an equal or greater amount of copies than the previous player did. If a player cannot (or does not want) to play, they pass until the next pizza starts.

At the end of the Shift, the new roles are distributed among the players according to the order of finishing, and every player gets the number of points related to their role.

The data were collected at several pilot tests that have been conducted recorded as videos. The qualitative analysis through observation of these data videos showed that the requirements 1-7 having been met. More specifically, we observed that the players mostly exhibited clear the intended emotional expression when the specially designed actions were invoked. Also, we observed that some players developed group-based strategies against a player who had a higher score.

\section{Next Steps: Involving the Robot as a Player}

The proposed game was designed to be used in an HRI scenario, in particular to 1) evoke natural and clear to recognize emotional reactions and social dynamics in the participants, 2) to enable and stimulate both the robot and the human players to form competitive strategies and 3) to enable a robot participating in the game to sense and monitor both the game status and the affective responses of each participant and the group as a whole.

The goal for the robot involved in the game will be to understand the affective behavior of the human participants and use this information, together with that of the game status, and to select the best strategy to play the game. The robot might either aim at winning the game or at selecting the behavior that maximizes a positive affective reaction in the other players. Previously, a human-robot game was developed in [13] in which an optimization algorithm was predicting the next 5 possible moves and choosing the optimal move based on this prediction. This made the robot always win it but did not consider the emotional state of the interacting child. To avoid negative emotions of the children, the researchers inverting the robot's winning strategies to make children always win [13] which is not an optimal strategy either. To cope with such problems, in the current Chef's Hat game, the choice of strategy is adaptive, and the adaptation will be based on the emotional state of the participants to provide richer possibilities for analyzing participants' emotions and play strategies. With this respect, the Chef's Hat game improves the social strategy of the game in addition to the game dynamics and logic-based strategies.

One of the most challenging problems we expect to face is the mechanical interaction of the robot with the card game. The envisioned robot that can get engaged in such an interaction is iCub which is a very advanced social robot, but still have limitations when interacting with real objects, in particular, with very thin objects such as cards. In this regard, we plan to adapt the way that the iCub interacts with the game. Our general idea is to have the iCub asking for help any time it needs to receive or withdraw a card, or put on and off a physical attribute at the beginning of the Shift and use the QR codes present in the card to generate an internal representation of which cards the robot has at hand. However, we are still evaluating the best way to make the robot place a card on the board during the game. Our current investigations involve weather by using a projector that will project the card of the robot on the board or using a specific cardholder that the robot can point at when it wants to place a card.

To achieve the socially intelligent and adaptive behavior that connects the learning of affective perception/display and game strategy, we envision a cognitive framework that will address the problem based on two directions. First, an adaptive affective perception, based on our previous work on artificial neural networks [2, 3], will use an unsupervised affective memory mechanism to overcome the problems of online learning. And second, an interactive reinforcement learning strategy based on learning the social and contextual impact of a certain action by measuring the affective responses of the players, avoiding the necessity of active human interaction in the learning loop.

\section{Conclusion}

In this paper, we proposed a new card game that makes it possible to systematically test the affective interactions and the development of competitive strategies between humans and robots with embodied intelligence. The developed game is based on research through a design approach and the game is specially designed to be used as a tool for gathering data and for improving the strategic and emotional interactions between humans and robots. The Chef's Hat card game has specially designed dynamics that integrate continual affective understanding into the game mechanics. These features were tested in several pilots during the design process. Also, the game was designed to easily incorporate learning algorithms that can be used to understand and produce strategic and affect-driven behavior, and therefore be a part of a platform for testing these behaviors (Figure 1). The robotic agents can be enabled to learn different action-selection strategies and optimize their gameplay style based on emotional interaction.

\section{REFERENCES}

[1] Emilia I Barakova, Roman Gorbunov, and Matthias Rauterberg. 2015. Automatic interpretation of affective facial expressions in the context of interpersonal interaction. IEEE transactions on human-machine systems 45,4 (2015), 409-418.

[2] Pablo Barros and Stefan Wermter. 2016. Developing crossmodal expression recognition based on a deep neural model. Adaptive behavior 24, 5 (2016), 373- 396.

[3] Pablo Barros and Stefan Wermter. 2017. A self-organizing model for affective memory. In Neural Networks (IJCNN), 2017 International Joint Conference on. IEEE, 31-38.

[4] Pablo Barros, Stefan Wermter, and Alessandra Sciutti. 2019. Towards Learning How to Properly Play UNO with the iCub Robot. arXiv preprint arXiv:1908.00744 (2019).

[5] Molly K Crossman, Alan E Kazdin, and Elizabeth R Kitt. 2018. The influence of a socially assistive robot on mood, anxiety, and arousal in children. Professional Psychology: Research and Practice 49, 1 (2018), 48. 
[6] Stan Franklin, Tamas Madl, Sidney DâĂŹmello, and Javier Snaider. 2013. LIDA: A systems-level architecture for cognition, emotion, and learning. IEEE Transactions on Autonomous Mental Development 6, 1 (2013), 19-41.

[7] Roman Gorbunov, Emilia I Barakova, and Matthias Rauterberg. 2017. Memory effect in expressed emotions during long term group interactions. In International Work-Conference on the Interplay Between Natural and Artificial Computation. Springer, 254-264.

[8] Masakazu Hirokawa, Atsushi Funahashi, Yasushi Itoh, and Kenji Suzuki. 2018. Adaptive behavior acquisition of a robot based on affective feedback and improvised teleoperation. IEEE Transactions on Cognitive and Developmental Systems (2018).

[9] Giorgio Metta, Giulio Sandini, David Vernon, Lorenzo Natale, and Francesco Nori. 2008. The iCub humanoid robot: an open platform for research in embodied cognition. In Proceedings of the 8th workshop on performance metrics for intelligent systems. ACM, 50-56.

[10] Ana Tanevska, Francesco Rea, Giulio Sandini, and Alessandra Sciutti. 2018. De- signing an Affective Cognitive Architecture for Human-Humanoid
Interaction. In Companion of the $2018 \mathrm{ACM} / \mathrm{IEEE}$ International Conference on Human-Robot Interaction. ACM, 253-254.

[11] Nguyen Tan Viet Tuyen, Sungmoon Jeong, and Nak Young Chong. 2018 Emotional Bodily Expressions for Culturally Competent Robots through Long Term Human-Robot Interaction. In 2018 IEEE/RSJ International Conference on Intelligent Robots and Systems (IROS). IEEE, 2008-2013.

[12] Greet Van de Perre, Hoang-Long Cao, Albert De Beir, Pablo Gómez Esteban, Dirk Lefeber, and Bram Vanderborght. 2018. Generic method for generating blended gestures and affective functional behaviors for social robots. Autonomous Robots 42, 3 (2018), 569-580.

[13] Emilia I. Barakova, Miriam De Haas, Wouter Kuijpers, Natalia Irigoyen, and Alessandro Betancourt. 2018. Socially grounded game strategy enhances bonding and perceived smartness of a humanoid robot, Connection Science, vol. 30 , no. 1, pp. 81-98.

[14] Roman Gorbunov, Emilia Barakova, and Matthias Rauterberg. 2013. Design of social agents. Neurocomputing, vol. 114, pp. 92-97. 